\title{
Article
}

\section{Diblock copolymer sphere morphology in ultra thin films under shear}

Pinna, Marco, Zvelindovsky, Andrei Victorovich, Guo, Xiaohu and Stokes, Christine L.

Available at http://clok.uclan.ac.uk/7445/

Pinna, Marco, Zvelindovsky, Andrei Victorovich ORCID: 0000-0003-4464-3254, Guo, Xiaohu and Stokes, Christine L. (2011) Diblock copolymer sphere morphology in ultra thin films under shear. Soft Matter, 7 (15). pp. 6991-6997. ISSN 1744-683X

It is advisable to refer to the publisher's version if you intend to cite from the work. http://dx.doi.org/10.1039/c1sm05478c

For more information about UCLan's research in this area go to http://www.uclan.ac.uk/researchgroups/ and search for <name of research Group>.

For information about Research generally at UCLan please go to http://www.uclan.ac.uk/research/

All outputs in CLoK are protected by Intellectual Property Rights law, including Copyright law. Copyright, IPR and Moral Rights for the works on this site are retained by the individual authors and/or other copyright owners. Terms and conditions for use of this material are defined in the policies page. 


\title{
Soft Matter
}

\section{Diblock copolymer sphere morphology in ultra thin films under shear}

\author{
Marco Pinna, ${ }^{* a b}$ Andrei V. M. Zvelindovsky, ${ }^{* a b}$ Xiaohu Guo ${ }^{c}$ and Christine L. Stokes ${ }^{a}$ \\ Received 19th March 2011, Accepted 4th May 2011 \\ DOI: $10.1039 / \mathrm{c} 1 \mathrm{sm} 05478 \mathrm{c}$
}

\begin{abstract}
We demonstrate that the shear alignment and the shear-induced transitions in sphere-forming diblock copolymer single layer and bilayer films observed experimentally [Y.-R Hong, D. H. Adamson, P. M. Chaikin and R. A. Register, Soft Matter, 2009, 5, 1687] can be explained by cell dynamics simulation, a simple model with a Ginzburg-Landau Hamiltonian. In two layer films the spheres align in various arrangements, like (100) or (110) bcc planes, or transform to cylinders depending on the shear rate and the temperature. For the first time, we present a nontrivial alignment mechanism of a single layer of spherical domains in shear via slug-like movement of transient cylindrical micelles. In addition, we clarify the formation of the perpendicular cylinders, found in the recent particle based simulation [A. Chremos, K. Margaritis, A. Z. Panagiotopoulos, Soft Matter, 2010, 6, 3588].
\end{abstract}

\section{Introduction}

A great challenge of soft nanotechnology is to achieve long range order of nanostructures in macroscopic size samples. Block copolymers are good candidates for this task due to their natural ability to self organize. ${ }^{1,2}$ Block copolymers are long chain molecules consisting of two or more chemically different blocks. Depending on their composition, molecular architecture and external factors, such as temperature, they can form various structures on the scale of their blocks with numerous potential applications. ${ }^{3}$ Thin films of block copolymers are of great interest for templating ordered patterns on surfaces. ${ }^{4}$ Therefore, tailoring of film structures is a very active area of the current research. External fields, such as electricic,5-7 and shear flow, ${ }^{1,5}$ are instruments often used for structure manipulation.

The current study was motivated by the need to understand the very nontrivial behaviour of the spherical morphology of block copolymers in ultra thin films under shear observed in recent experiments. ${ }^{8}$ Shearing of spherical bulk phase and shear induced sphere-to-cylinder transition in bulk have a long history. ${ }^{9-11}$ Observing that phenomena in thin films, which are much more attractive from an application point of view, has only become possible very recently. ${ }^{8,12-15}$ Not only can spheres transform to cylinders above a certain shear rate, but one can achieve a perfect alignment of spheres in layers at lower shear rates. ${ }^{8}$ Initially, it was suggested that two or more layers are required for such an alignment, ${ }^{12}$ while, most recently, a single layer film was successfully aligned by shear. ${ }^{8}$ A possible mechanism for the

${ }^{a}$ Computational Physics Group, University of Central Lancashire, Preston, PR12HE, United Kingdom.E-mail: MPinna@uclan.ac.uk

${ }^{b}$ Institute for Nanotechnology and Bioengineering, University of Central Lancashire, Preston, PRI 2HE, United Kingdom

'Advanced Research Computing Group, Daresbury Laboratory, Warrington, WA4 4AD, United Kingdom latter phenomenon was suggested in ref. 8 inspired by our earlier simulations for the bulk sphere system. ${ }^{16}$ A confirmation of that suggestion was required, together with a complete picture of how such an alignment occurs in time, both in single layer and two layer systems.

Computer simulations of sheared block copolymer systems also have a long history (see review ${ }^{\mathbf{1 7}}$ ), thus we focus here only on the works dealing with the sphere morphology. Dynamic density functional theory simulation of bulk sphere systems described both sphere-to-cylinder transition and survival of spheres under shear in the form of elongated ellipsoids. ${ }^{16}$ Cell dynamics simulation (CDS) of larger simulation boxes confirmed these findings and also found alignment of spheres in hexagonally packed sliding layers. ${ }^{18}$ Molecular dynamics simulation of a bilayer film revealed shear-induced ordering of hexagonally arranged spheres in a shear flow. ${ }^{19}$ Most recently, a particle-based Langevin dynamics simulation found shear-induced transition of spheres to cylinders ${ }^{20}$ (ref. 20 presents a study for a wide range of the composition parameter $f$ encompassing sphere-forming and not sphere-forming diblock copolymers). Although the spheres survived in a shear flow below critical values of the shear rate, no alignment of the hexagonal lattice in a shear was reported for a single layer system. ${ }^{20}$ Particle based simulations ${ }^{19,20}$ are limited to relatively small box sizes, which do not allow the study of kinetics on the scale of grains of nanostructures. One of the solutions to study very large systems is to model spheres as nondeformable particles in, for instance, Brownian dynamics simulation. ${ }^{19,21}$ That is, however, more appropriate for colloidal crystals, as such a model would not be able to capture both phenomena: alignment of spheres and morphological transition to cylinders in a shear flow. Another option is to apply the cell dynamics simulation, which is widely used to describe mesoscopic structure formation in diblock copolymer systems. ${ }^{22-32}$ CDS has a record of remarkable agreement with experiments on 
sphere-forming block copolymers. ${ }^{33}$ However, it should be noted that as CDS is a coarse-grained field method it cannot capture fine details accessible to particle based methods. For instance, in the simulation study in ref. 20 phase separation is introduced into the system via attractive interactions between the beads composing the minority blocks (all other interactions are purely repulsive). This asymmetry in the interaction could result in different behaviour (e.g. different local viscosities) relative to the symmetric interactions assumed in the CDS model. Therefore, a direct comparison between the two approaches is not possible at the moment and an analogy between them can only be drawn on generic features of the system behaviour.

In the present work we employ CDS to study ultra-thin block copolymer films of one and two sphere layers under shear. Our results complement the experimental findings ${ }^{8}$ by providing details of the time evolution of the structures in large simulation boxes, reaching the sample sizes relevant to the experiments. Our results unveil many nontrivial features, which were not possible to see by other simulation methods. For instance, the alignment in a single layer was found to have an additional kinetic stage compared to the initially suggested mechanism. ${ }^{8}$

\section{Simulation method}

In the cell dynamics simulation an order parameter $\psi(\mathbf{r}, t)$ of an $\mathrm{AB}$ diblock copolymer melt is determined as

$$
\psi=\varphi_{\mathrm{A}}-\varphi_{\mathrm{B}}+(1-2 f)
$$

where $\varphi_{\mathrm{A}}$ and $\varphi_{\mathrm{B}}$ are the local volume fractions of $\mathrm{A}$ and $\mathrm{B}$ monomers respectively, and $f$ is the volume fraction of $\mathrm{A}$ monomers in the diblock, $f=N_{\mathrm{A}} /\left(N_{\mathrm{A}}+N_{\mathrm{B}}\right)$ with $N_{i}$ being the number of monomers in the $i$-block.

The time evolution of the order parameter is given by the Cahn-Hilliard-Cook (CHC) equation: ${ }^{24,25}$

$$
\frac{\partial \psi}{\partial t}=M \nabla^{2}\left(\frac{\delta F[\psi]}{\delta \psi}\right)+\eta \xi(\mathbf{r}, t)
$$

where $M$ is a phenomenological mobility constant. Here we set $M=1$, which correspondingly sets the timescale for the diffusive processes (the dimensionless time is $t M / a_{0}^{2}$, where the lattice cell size $a_{0}$ is set to 1 ). The last term in eqn (2) is a noise term where $\eta$ is the amplitude of the noise and $\xi(\mathbf{r}, t)$ is a normalised Gaussian random noise, which satisfies the fluctuation-dissipation theorem. $F[\psi]$ is the free energy functional divided by $k T$, which can be written as: ${ }^{23}$

$$
\begin{aligned}
& F[\psi(\mathbf{r})]=\int \mathrm{d} \mathbf{r}\left[H(\psi)+\frac{D}{2}|\nabla \psi|^{2}\right] \\
& +\frac{B}{2} \int \mathrm{d} \mathbf{r} \int \mathrm{d} \mathbf{r}^{\prime} G\left(\mathbf{r}-\mathbf{r}^{\prime}\right) \psi(\mathbf{r}) \psi\left(\mathbf{r}^{\prime}\right)
\end{aligned}
$$

where the first and second terms are the short and the long-range interaction terms, respectively, the coefficient $D$ is a positive constant that plays the role of a diffusion coefficient, the Green function $G\left(\mathbf{r}-\mathbf{r}^{\prime}\right)$ for the Laplace equation satisfies $\nabla^{2} G\left(\mathbf{r}-\mathbf{r}^{\prime}\right)=-\delta\left(\mathbf{r}-\mathbf{r}^{\prime}\right), B$ is a parameter that introduces a chainlength dependence to the free-energy, ${ }^{26}$ and $H(\psi)$ is the free energy: ${ }^{26,27}$

$$
H(\psi)=\left[-\frac{\tau}{2}+\frac{A}{2}(1-2 f)^{2}\right] \psi^{2}+\frac{v}{3}(1-2 f) \psi^{3}+\frac{u}{4} \psi^{4}
$$

Here $\tau$ is a temperature parameter and $A, v, u$ are phenomenological constants. ${ }^{27}$ All these parameters can be related to molecular characteristics. According to Ohta and Kawasaki ${ }^{34}$ $\tau^{\prime}=-\tau+A(1-2 f)^{2}, D$, and $B$ can be expressed in terms of degree of polymerisation $N$, the segment length $b$ and the Flory-Huggins parameter $\chi$ (which is inversely proportional to temperature):

$$
\begin{gathered}
\tau^{\prime}=-\frac{1}{2 N}\left(N \chi-\frac{s(f)}{4 f^{2}(1-f)^{2}}\right), \quad D=\frac{b^{2}}{48 f(1-f)}, \\
B=\frac{9}{4 N^{2} b^{2} f^{2}(1-f)^{2}}
\end{gathered}
$$

where $s(f)$ is an empirical fitting function of the order of 1 (e.g. $s(0.5)=0.9, s(0.3)=1.0) .{ }^{34}$ In simulation we use dimensionless parameters $\tilde{D}=D / a_{0}^{2}$ and $\tilde{B}=B a_{0}^{2}$ (for simplicity we keep notations $D$ and $B$ instead of $\tilde{D}$ and $\tilde{B}$ ). The parameters $u$ and $v$ do not allow for a compact representation and can be computed by evaluating the appropriate vertex function given by Leibler. ${ }^{35}$ These are very complex functions that can be only approximately replaced by constants. We believe that the phenomenology we are studying is quite general and, hence, we allow the freedom of choosing the parameters in eqn (2)-(4) as phenomenological constants.

In the case of the flow $\mathbf{v}=\left(v_{x}, v_{y}, v_{z}\right)$ eqn (2) becomes: ${ }^{23,24}$

$$
\frac{\partial \psi}{\partial t}+\nabla \cdot(\mathbf{v} \psi)=M \nabla^{2}\left(\frac{\delta F[\psi]}{\delta \psi}\right)+\eta \xi(\mathbf{r}, t)
$$

We consider a steady shear flow defined by: $v_{x}=\dot{\gamma} y, \quad v_{y}=v_{z}=0$. Thus the numerical evolution of eqn (6) is given by: ${ }^{29,32}$

$$
\begin{aligned}
& \psi(\mathbf{n}, t+1)=\psi(\mathbf{n}, t)-\{\langle\langle\Gamma(\mathbf{n}, t)\rangle\rangle-\Gamma(\mathbf{n}, t)+B \psi(\mathbf{n}, t)-\eta \xi(\mathbf{n}, t) \\
& \left.+\frac{1}{2} \tilde{\dot{\gamma}} \tilde{y}\left[\psi\left(n_{x}+1, n_{y}, n_{z}, t\right)-\psi\left(n_{x}-1, n_{y}, n_{z}, t\right)\right]\right\}
\end{aligned}
$$

where $\mathbf{n}=\left(n_{x}, n_{y}, n_{z}\right)$ is the position on the cubic grid $L_{x} \times L_{y} \times$ $L_{z}$, and $\langle\langle X\rangle\rangle$ is given by: ${ }^{22}$

$$
\langle\langle X\rangle\rangle=\frac{6}{80} \sum_{\mathrm{NN}} X+\frac{3}{80} \sum_{\mathrm{NNN}} X+\frac{1}{80} \sum_{\mathrm{NNNN}} X
$$

to calculate the isotropized Laplacian $\langle\langle X\rangle\rangle-X$. The dimensionless shear rate is $\tilde{\dot{\gamma}}=\dot{\gamma} a_{0}^{2} / M$, the dimensionless coordinate is $\tilde{y}=y / a_{0}$ (we will use the notation $y$ instead of $\tilde{y}$ ) and

$$
\Gamma(\mathbf{n}, t)=g(\psi(\mathbf{n}, t))-\psi(\mathbf{n}, t)+D[\langle\langle\psi(\mathbf{n}, t)\rangle\rangle-\psi(\mathbf{n}, t)]
$$

where the so-called map function is: ${ }^{23,27}$

$$
g(\psi)=\left[1+\tau-A(1-2 f)^{2}\right] \psi-v(1-2 f) \psi^{2}-u \psi^{3}
$$

In the case of a confinement between two walls placed at $n_{y}=1$ and $n_{y}=L_{y}$ the algorithm, eqn (9), has to be modified: ${ }^{.32}$

$\Gamma(\mathbf{n}, t)=g(\psi(\mathbf{n}, t))-\psi(\mathbf{n}, t)+D[\langle\langle\psi(\mathbf{n}, t)\rangle\rangle-\psi(\mathbf{n}, t)]-s_{i}(y)(11)$ 
where

$$
s_{i}(y)=h_{i} \cdot \varphi_{i} \cdot \delta_{n_{y}=1} \text { or } n_{y}=L_{y}
$$

$i$ denoting the segment of the block copolymer $(A$ or $B),{ }^{32} h_{i}$ the strength of the interaction between the walls and the segments, and $\delta_{a=b}$ the Kronecker delta. Instead of sheared periodic boundary conditions used for bulk systems, ${ }^{36}$ for our geometry of confinement and shear we use reflective boundary conditions in the $y$-direction and periodic boundary conditions in the $x$ - and $z$-directions.

CDS is reasonably fast and can be performed in relatively large boxes. However, in order to link simulation results with experiments it is necessary to use very large simulation boxes, which cannot be achieved even with this method on modern single processor computers. Therefore, in our work we use a highly scalable parallel implementation of CDS. ${ }^{37}$

\section{Results}

\section{A. Model system}

Our system of interest is a sphere forming diblock copolymer melt. We use parameters for such a system from our previous work: ${ }^{18} f=0.4, u=0.38, v=2.3, B=0.01, D=0.5, A=1.5$. A similar sphere forming system (only different in $D=0.2$ ) was studied in ref. 27. The composition parameter $f$ in our study is different from the experimental system ${ }^{8}$ due to the choice of the numerical CDS model. The CDS scheme is known to work best when the composition parameter $f$ is not too small. ${ }^{27}$ Here we do not attempt to mimic a specific experimental system but we are aiming to study the generic behaviour of a sphere forming system that is well investigated in the literature. Using eqn (5) one can obtain the degree of polymerisation for our system, $N=26$. The focus of the present paper is on the ultra thin films accommodating a double or single layer of spheres. By performing a series of simulations for different film thickness $L_{y}$ and different values for the boundary condition on the film surfaces $h$, eqn (12), we determined the best parameters to represent a single and two layers systems: $\left\{H=20, h_{\mathrm{A}}=0.2\right\}$ and $\left\{H=26, h_{\mathrm{A}}=0.2\right\}$, respectively. In order to eliminate a possible box size effect on our conclusions, we counter-checked our simulations for two lateral sizes $L_{x}=L_{z}=128$ and 512, and we present the results for the largest lateral box size $L_{x}=L_{z}=512$.

\section{B. Bilayer films}

Following the experimental work, ${ }^{8}$ we first present the results for the films accommodating two layers of spheres.

Initially, prior to the application of shear, the structures were equilibrated for 1000000 timesteps. We systematically vary the temperature parameter $\tau$ and the shear rate $\tilde{\dot{\gamma}}$. The topography of the top layer of the film demonstrates a rather complex behaviour shown in Fig. 1. When the system is closer to the orderdisorder transition (ODT), at the lower values of $\tau$, we observe an interesting arrangement of spheres at very low shear rates, that is multiple domains of spheres on a square lattice, which corresponds to the (100)-plane of the body-centred cubic (bcc) morphology ( $\tau=0.15$, symbol * in Fig. 1). At the same low shear rates, but further away from the ODT ( $\tau=0.20, \bullet$ in Fig. 1) the system arranges itself in multiple domains of hexagonally packed spheres which corresponds to the (110)-plane or bcc morphology with no global in-plane order. ${ }^{38}$ We consider several lines in the phase diagram in Fig. 1 in a more detail.

At low $\tau=0.15$ (closer to the ODT) the multiple square lattice transforms into a single square domain upon increase of shear rate (Fig. $1 \bigcirc$ ). With further increasing of the shear rate, the symmetry of the lattice changes from the square to the hexagonal monocrystal (Fig. 1 a). The sequence of morphologies is different further away from the ODT. For instance, the case of $\tau=0.20$ is shown in more detail in Fig. 2 and 3. At low shear rates we do not observe a square arrangement of spheres, instead we have multiple clusters of hexagonally packed spheres. With the increase of shear the number of clusters decreases, and on the defect lines, such as grain boundaries, the spheres merge into the long-living cylinders, which move perpendicularly to the flow direction (see Fig. $1 \mathbf{\Lambda}$, 2 and 3). Perpendicular to the flow, cylinders were found earlier by a particle based simulation in refs 20 and 39, albeit for different systems: ref. 20 reports perpendicular cylinders for cylinderforming diblock copolymer melts and ref. 39 reports a "log-rolling" cylindrical phase for a symmetric diblock copolymer in a selective solvent. Simulation boxes in the works ${ }^{20,39}$ accommodated much smaller systems compared to our work, and therefore such stabilisation of perpendicular cylinders could be, in principle, due to a subtle effect of the box boundaries. In our simulation boxes we observe that such perpendicular cylinders are stabilised by the boundaries between the grains of the hexagonally packed spheres. We note, however, that the simulation model used in refs 20 and 39 has a more complete description of the shear flow and therefore a direct analogy between our methods is not possible. It should be noted that for cylinder-forming systems "log-rolling" was reported in several experimental studies..$^{40-42}$

At higher shear rates, the system forms a nearly perfect monocluster of hexagonally packed spheres (Fig. $1 \mathbf{m}, 2$ and 3). The reason behind such a perfect arrangement can be seen in Fig. 4 . Although both top and bottom layer structures generally follow each other (see Fig. 2 and 3), they are necessarily shifted due to the geometry of the bcc morphologies (Fig. 4). Therefore, each layer of spheres provides sliding guides to another layer (Fig. 4a and $b$ ). This picture supports the schematic mechanism proposed in the experimental work ref. 12 and observed by a particle based simulation in ref. 19. It also explains the orientation of the hexagon in the shear flow, as one which allows for the most 'comfortable' sliding of spheres due to a larger distance between rows of spheres. Such sliding helps to remove defects as shear proceeds. A typical example is shown in Fig. 4c. One layer of spheres (seen as black circles) is already perfectly hexagonal, while the other (grey spheres) has characteristic defects in the forms of 5-7 sphere pairs. In these places the spheres from different layers superimpose and roll over each other in a flow (indicated by dashed arrows in Fig. 4c). That deforms the spheres and eventually eliminates the defects. Fig. $4 d$ shows the Euler characteristic (one of the Minkowski functionals ${ }^{18}$ ), which in this case is equal to the number of spheres. We observe that as the shear proceeds, the overall number of spheres slightly drops, analogous to what we observed before for the sheared spheres in the bulk. ${ }^{18}$ Therefore, there is a similarity between the defect annihilation kinetics in the films of two layers and in the two neighbouring layers in the bulk. 


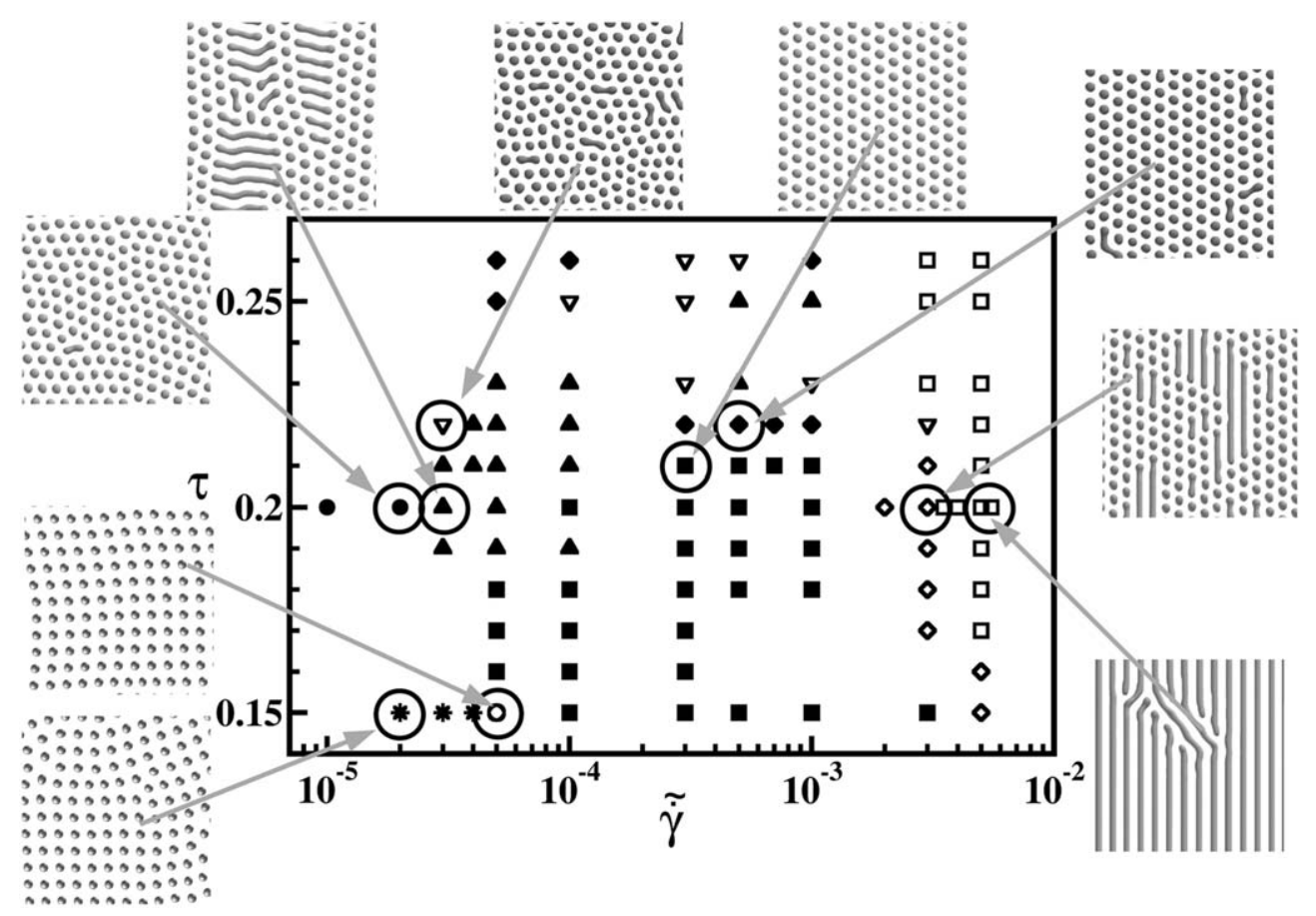

Fig. 1 A morphology diagram of the top layer of a bilayer film as a function of the temperature parameter $\tau$ and the shear rate $\tilde{\dot{\gamma}}$. Typical morphologies are illustrated by crops of the larger simulation boxes. Encircled symbols represent the actual simulation of the shown crops. The shear direction is vertical in all images.
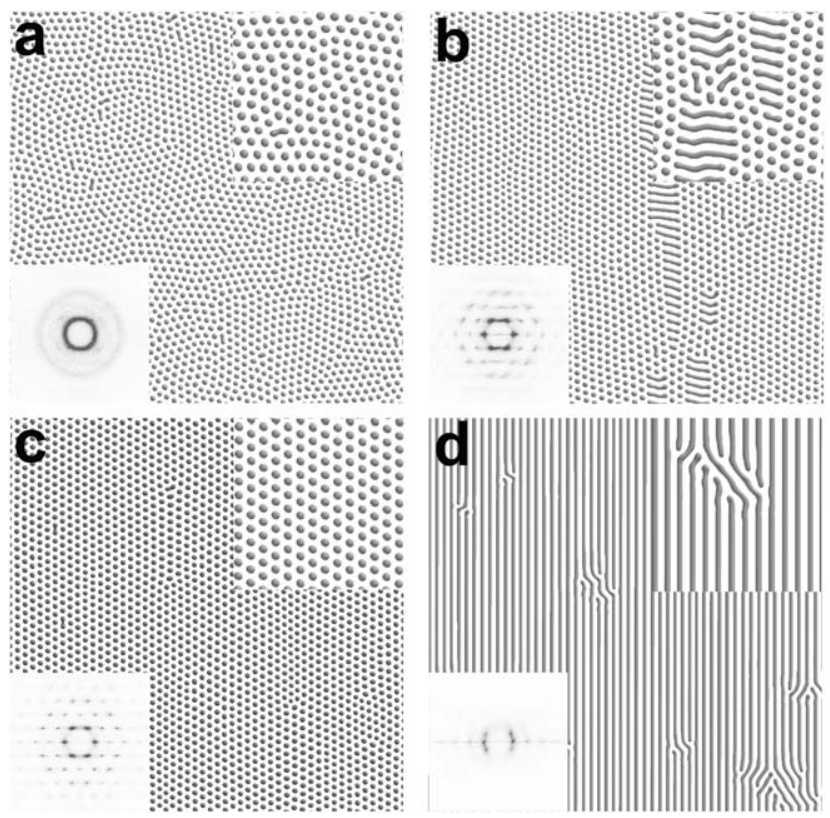

Fig. 2 Morphologies of the top layer of a bilayer film for $\tau=0.20$ and different shear rates: $\tilde{\dot{\gamma}}=2 \times 10^{-5}$ (a), $5 \times 10^{-5}$ (b), $3 \times 10^{-4}$ (c), $5 \times 10^{-3}$ (d). Insets show associated FFTs of the images and enlarged crops of the simulation boxes. The shear direction is vertical in all images.

At even higher shear rates the spheres transform to cylinders (Fig. $1 \square, 2 \mathrm{~d}$ and 3d). This transformation occurs via deforming spheres into ellipsoids and eventual merging. Coexistence of elongated spheres and short cylinders can be observed at intermediate shear rates $($ Fig. $1 \diamond$ ). Such sphere-cylinder coexistence
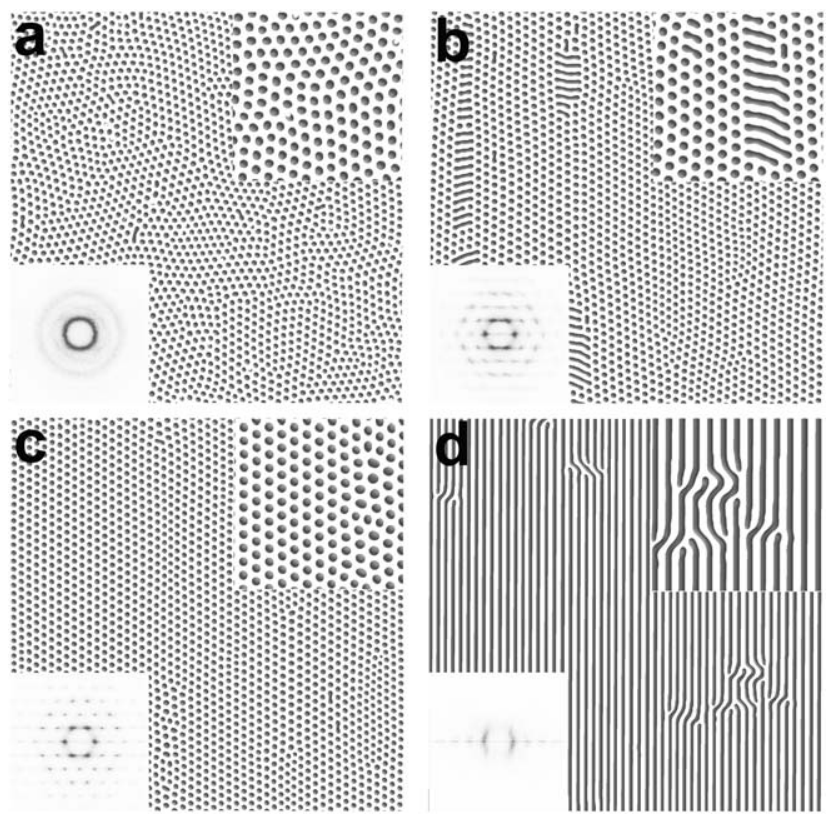

Fig. 3 Morphologies of the lower layer of the bilayer film from Fig. 2. Insets show associated FFTs of the images and enlarged crops of the simulation boxes. The shear direction is vertical in all images.

under shear was also observed in particle based simulations in ref. 20.

The temperature dependence of this transition can be deduced by comparing results for different $\tau$. For instance, for $\tilde{\gamma}=0.0003$ the system exhibits perfect hexagonal packing of spheres at low $\tau$, defected hexagonal packing (with short parallel and 

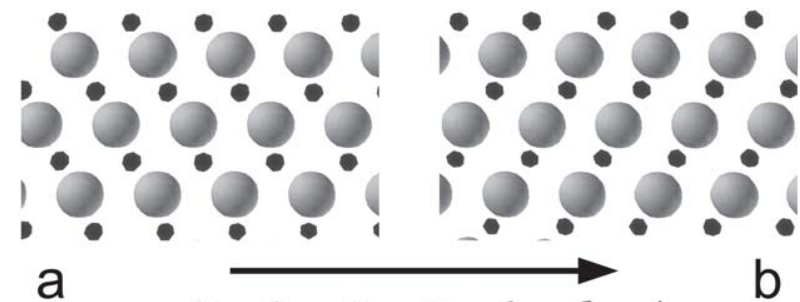

a
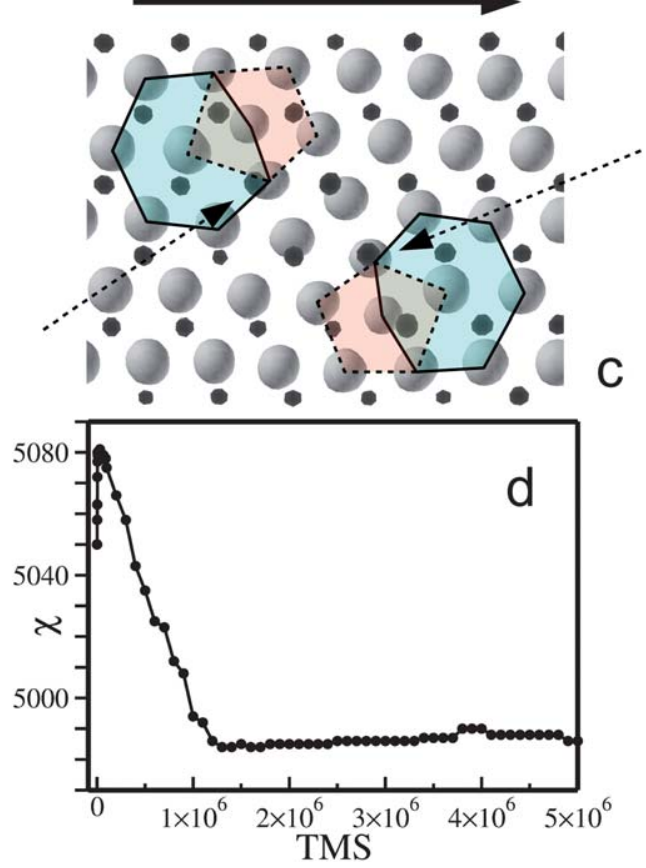

Fig. 4 The defect removal mechanism in a bilayer film for $\tau=0.18$ and $\tilde{\dot{\gamma}}=3 \times 10^{-4}$. Crops of the larger simulation boxes at different times: 1.1 $\times 10^{6}(\mathrm{a})$ and $1.6 \times 10^{6}(\mathrm{~b})$ timesteps. The horizontal solid arrow indicates the direction of the shear flow. One layer of spheres is shown in full, while the second layer is cut for visualisation purposes so only the tops of spheres are seen as black circles. (c) A different crop of the simulation snapshot at $1.6 \times 10^{6}$ timesteps. Two 5-7 pairs of spheres are shown by polygons. Overlapping spheres in different layers are indicated by dashed arrows. (d) Euler characteristic (number of spheres) as a function of timestep (TMS).

perpendicular cylinders, Fig. $1 \bullet$ ) at higher $\tau$, and disordered spheres with randomly oriented short cylinders (Fig. $1 \nabla$ ). These disordered spheres produce a ring in a fast Fourier transform (FFT). As $\tau$ is inversely proportional to the temperature, the observed behaviour is similar to the experimental situation (compare six-spot and circle FFTs for $150^{\circ} \mathrm{C}$ and $140^{\circ} \mathrm{C}$ at $5 \mathrm{kPa}$ in Fig. 2 of ref. 8).

In the experimental work $^{8}$ some of the patterns are slightly tilted with respect to the shear flow. That could be due to the sample preparation techniques, however, the possibility of intermediate orientations cannot be ruled out. Our results show that in the large parameter space (Fig. 1 a), the mono-cluster of hexagonally packed spheres is aligned perfectly with the flow direction, as shown in Fig. 4. However, at low $\tau$ and not high shear rates a titled hexagon orientation was also found to be stable within the simulation time (Fig. 5).

Although morphologies of both top and bottom layers generally follow each other in our simulations (see Fig. 2 and 3), in agreement with conclusions from the experiments in ref. 8 ,

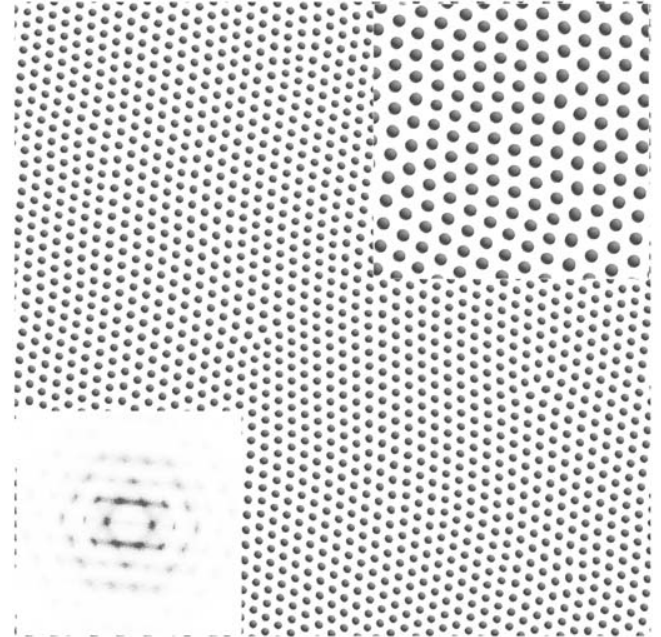

Fig. 5 The tilted orientation of morphology for $\tau=0.16$ and $\tilde{\dot{\gamma}}=5 \times 10^{-5}$. Insets show associated FFT of the image and an enlarged crop of the simulation box. The shear direction is vertical.

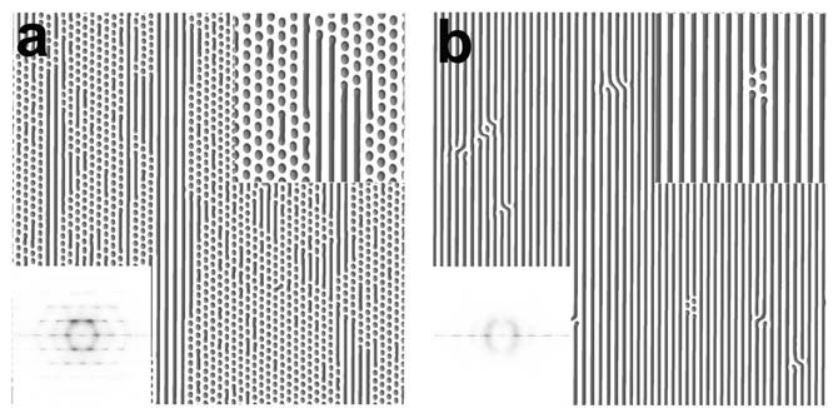

Fig. 6 Different morphologies of the bottom (a) and top (b) layers for $\tau=0.20$ and $\tilde{\dot{\gamma}}=3.5 \times 10^{-3}$. Insets show associated FFTs of the images and enlarged crops of the simulation boxes. The shear direction is vertical in all images.

they can differ close to a phase boundary (see Fig. 6). While the top layer is already transformed to cylinders, the bottom layer remains in a coexistence of mostly elongated ellipsoids and some

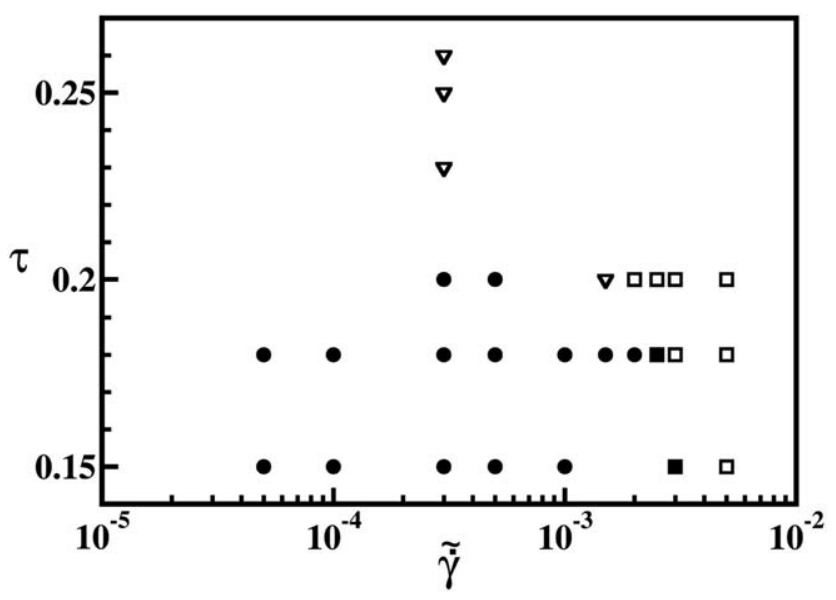

Fig. 7 A morphology diagram of a single-layer film as a function of the temperature parameter $\tau$ and the shear rate $\tilde{\dot{\gamma}}$. The symbols depict the same morphologies as shown in Fig. 1. 


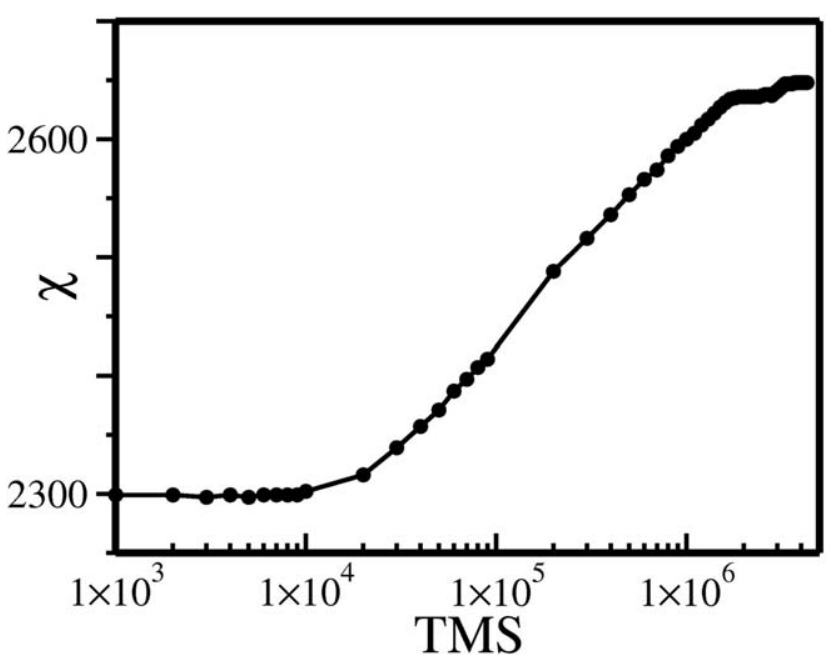

Fig. 8 Euler characteristic (number of spheres) of a single layer system for $\tau=0.15$ and $\tilde{\dot{\gamma}}=3 \times 10^{-3}$ as a function of timestep (TMS).

cylinders. This situation is rather similar to the experimental images in Fig. 1c and 3c in ref. 8, although the authors attribute the "broken" structure in the bottom layer to the etching process.

\section{Single-layer films}

Fig. 7 shows results for shearing of a single layer of spheres. Above a certain value of the shear rate, the spheres transform to cylinders similar to the situation for the two layer system (see Fig. $1 \square$ ). At higher values of $\tau$ we find the disordered spheres similar to the two layer system (open triangles in Fig. 1 and 7). Transition to cylinders in a single layer of spheres was also found experimentally in ref. 8 and by a particle based simulation in ref. 20. The difference between the single and the two layer systems is that the region in the morphology diagram occupied by multiple hexagonal clusters without any global orientation is much larger for the single layer system compared to the bi-layer system (symbol $\bigcirc$ in Fig. 1 and 7). In the experimental work in ref. 12, the authors conclude that in order to achieve a shear alignment of spheres, two or more layers are required. However, in a more recent experimental work ${ }^{8}$ the authors managed to align a single layer of spheres for a different copolymer system. In the simulations in ref. 20, which were performed for a single layer of spheres, the authors do not report achieving shear alignment, moreover, their spherical system images do not exhibit evident hexagonal packing due to many defects. In our simulations we find that such an alignment can also be achieved in a single layer (Fig. 7 a). Fig. 8 shows the Euler characteristic evolution for such shear alignment. The Euler characteristic, which is equal to the number of spheres, exponentially increases in time until it reaches a plateau. This behaviour is very different from the bilayer system, where the number of spheres slightly drops (Fig. 4d). That indicates a totally different alignment mechanism. The authors of the experimental work $^{8}$ propose that if the spheres are deformed into ellipsoids by the shear, this would break the symmetry and provide a mechanism for shear alignment, even in single-layer films. However, in our simulations the deformation to ellipsoids alone does not produce the alignment by itself, which is the reason for a very large region of not aligned spheres in the morphology diagram (Fig. 7 ). Our simulations unveil a very nontrivial kinetic mechanism of how the alignment proceeds in time (Fig. 9). Prior to the application of the shear, the system consist of multiple hexagonal clusters (Fig. 9a), when the shear is applied it deforms the spheres into ellipsoids, which
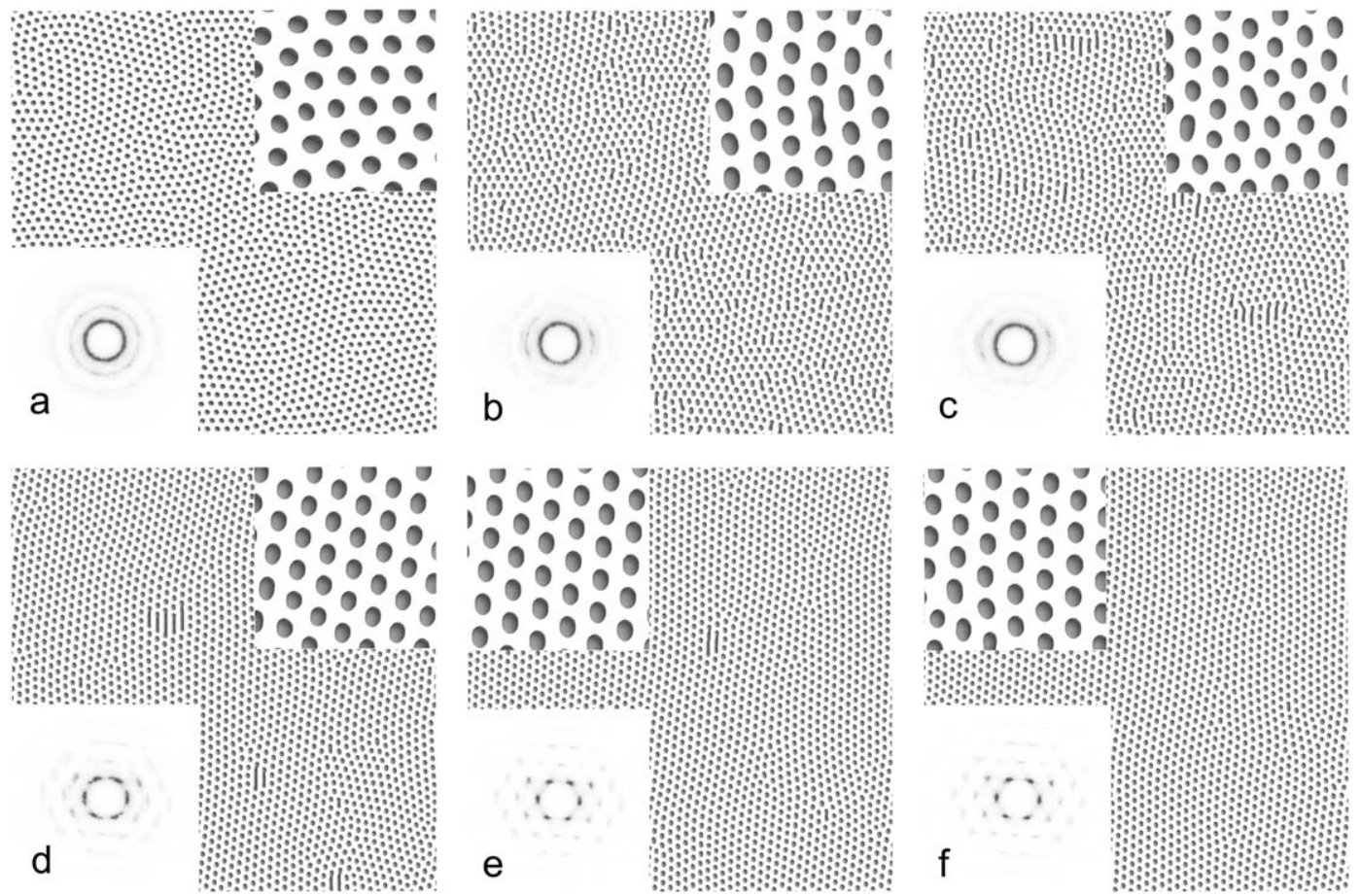

Fig. 9 Time evolution of the alignment process for a single layer system for $\tau=0.15$ and $\tilde{\dot{\gamma}}=3 \times 10^{-3}$ at different timesteps: 0 (a), $2 \times 10^{4}$ (b), $10^{5}$ (c), $10^{6}(\mathrm{~d}), 2.8 \times 10^{6}(\mathrm{e}), 4.3 \times 10^{6}(\mathrm{f})$. The shear direction is vertical in all images. 
occasionally merge into short cylinders of 2-4 domain spacings long (worm-like micelles in Fig. 9b, c). These cylinders move in the shear direction as slugs leaving behind a pearl-necklace trail of aligned spheres (Fig. 9b, c). As the pattern becomes more and more aligned, the number of cylinders decreases as they split back into spheres (Fig. 9e). At the end, all cylinders disappear, leaving a perfectly aligned mono-cluster of hexagonally packed ellipsoids (Fig. 9f).

\section{Conclusions}

Cell dynamics simulations have been used to gain insight on sheared sphere-forming diblock copolymer systems in ultra thin films. The study was mainly motivated by the recent experiments on such systems. ${ }^{8}$

Our simulations are performed in large simulations boxes comparable in size with the experimental images. Our findings capture a very rich shear behaviour and structures, including multiple and mono-cluster square packing, multiple and monocluster hexagonal packing, hexagonal packing with cylindrical impurities, disordered spheres packing, perpendicular and parallel to the flow cylinders, ellipsoids, as well as coexistence of ellipsoids and cylinders. Several of these behaviours are observed experimentally. ${ }^{8}$ We demonstrate the mechanism of sphere alignment in bi-layer system via spheres sliding in neighbouring layers. We elaborate on the sphere-to-cylinder transition under shear. In addition to experimentally observed parallel cylinders, we obtain perpendicular cylinders forming on grain boundaries, which complements recent particle based simulation of smaller (in domain spacings) systems. ${ }^{20} \mathrm{We}$ study the same phenomena in single layer systems as well. Although the deformation of spheres into ellipsoids was already observed in bulk simulations, ${ }^{\mathbf{1 6}, 18}$ the kinetic mechanism of alignment via slug-like movement of transient worm-like micelles does not occur in bulk simulations and is intrinsic to single layer films. To the best knowledge of the authors this is the first time a simulation explains sphere alignment in a single layer thin film, and the first time when all these complex behaviours in one vs. two layers systems have been observed by a single simulation method.

\section{Acknowledgements}

MP and AVMZ acknowledge the support of the EU project MEMORY within NanoSci-E+ ERA-NET Plus Action (EPSRC, EP/H006583/1). MP and CS thank the Nuffield Foundation for the summer internship grant. CS acknowledges the generous support from the UCLan's Centre for ResearchInformed Teaching.

The simulations were performed on Altix 3700 at UCLan HPC Facility.

\section{References}

1 I. W. Hamley. The Physics of Block Copolymers, Oxford University Press, Oxford, 1998.

2 Nanostructured Soft Matter, ed. A. V. Zvelindovsky, Springer, Dordrecht, 2007.
3 M. J. Fasolka and A. M. Mayes, Annu. Rev. Mater. Res., 2001, 31, 323.

4 S. Park, D. H. Lee, J. Xu, B. Kim, S. W. Hong, U. Jeong, T. Xu and T. P. Russell, Science, 2009, 323, 1030-1033.

5 T. Xu, J. T. Goldbach and T. P. Russell, Macromolecules, 2003, 36, 7296-7300.

6 T. L. Morkved, M. Lu, A. M. Urbas, E. E. Ehrichs, H. M. Jaeger, P. Mansky and T. P. Russell, Science, 1996, 273, 931.

7 V. Olszowka, L. Tsarkova and A. Böker, Soft Matter, 2009, 5, 812819.

8 Y.-R. Hong, D. H. Adamson, P. M. Chaikin and R. A. Register, Soft Matter, 2009, 5, 1687-1691.

9 K. A. Koppi, M. Tirrell, F. S. Bates, K. Almdal and K. Mortensen, J. Rheol., 1994, 38, 999.

10 I. W. Hamley, J. A. Pople, J. P. A. Fairclough, N. J. Terrill, A. J. Ryan, C. Booth, G.-E. Yu, O. Diat, K. Almdal, K. Mortensen and M. Vigild, J. Chem. Phys., 1998, 108, 6929.

11 C. Park, S. Simmons, L. J. Fetters, B. Hsiao, F. Yeh and E. L. Thomas, Polymer, 2000, 41, 2971.

12 D. E. Angelescu, J. H. Waller, R. A. Register and P. M. Chaikin, $A d v$. Mater., 2005, 17, 1878-1881.

13 M. W. Wu, R. A. Register and P. M. Chaikin, Phys. Rev. E: Stat., Nonlinear, Soft Matter Phys., 2006, 74, 040801(R).

14 A. P. Marencic, M. W. Wu and R. A. Register, Macromolecules, 2007, 40, 7299-7305.

15 A. P. Marencic, D. H. Adamson, P. M. Chaikin and R. A. Register, Phys. Rev. E: Stat., Nonlinear, Soft Matter Phys., 2010, 81, 011503.

16 A. V. M. Zvelindovsky and G. J. A. Sevink, Europhys. Lett., 2003, 62, 370-376.

17 I. Rychkov, Macromol. Theory Simul., 2005, 207, 14.

18 M. Pinna, A. V. Zvelindovsky, S. Todd and G. Goldbeck-Wood, J. Chem. Phys., 2006, 125, 154905.

19 G. Arya, J. Rottler, A. Z. Panagiotopoulos, D. J. Srolovitz and P. M. Chaikin, Langmuir, 2005, 21, 11518-11527.

20 A. Chremos, K. Margaritis and A. Z. Panagiotopoulos, Soft Matter, 2010, 6, 3588-3595.

21 J. Rottler and D. J. Srolovitz, Phys. Rev. Lett., 2007, 98, 175503.

22 Y. Oono and S. Puri, Phys. Rev. A: At., Mol., Opt. Phys., 1988, 38, 434.

23 M. Bahiana and Y. Oono, Phys. Rev. A: At., Mol., Opt. Phys., 1990, 41, 6763.

24 T. Ohta, Y. Enomoto, J. L. Harden and M. Doi, Macromolecules, 1993, 26, 4928.

25 H. Kodama and M. Doi, Macromolecules, 1996, 29, 2652.

26 I. W. Hamley, Macromol. Theory Simul., 2000, 9, 363.

27 S. R. Ren and I. W. Hamley, Macromolecules, 2001, 34, 116.

28 S. R. Ren, I. W. Hamley, P. I. C. Teixeira and P. D. Olmsted, Phys. Rev. E: Stat. Phys., Plasmas, Fluids, Relat. Interdiscip. Top., 2001, 63, 041503.

29 S. R. Ren, I. W. Hamley, G. J. A. Sevink, A. V. Zvelindovsky and J. G. E. M. Fraaije, Macromol. Theory Simul., 2002, 11, 123.

30 J. Feng, H. Liu and Y. Hu, Macromol. Theory Simul., 2002, 11, 549.

31 J. Feng, H. Liu and Y. Hu, Macromol. Theory Simul., 2002, 11, 556.

32 J. Feng and E. Ruckenstein, J. Chem. Phys., 2004, 121, 1609.

33 D. A. Vega, C. K. Harrison, D. E. Angelescu, M. L. Trawick, D. A. Huse, P. M. Chaikin and R. A. Register, Phys. Rev. E: Stat., Nonlinear, Soft Matter Phys., 2005, 71, 061803.

34 T. Ohta and K. Kawasaki, Macromolecules, 1986, 19, 2621.

35 L. Leibler, Macromolecules, 1980, 13, 1602.

36 M. Doi and D. Chen, J. Chem. Phys., 1989, 70, 5271.

37 X. Guo, M. Pinna and A. V. Zvelindovsky, Macromol. Theory Simul., 2007, 16, 779-784.

38 G. E. Stein, E. W. Cochran, K. Katsov, G. H. Fredrickson, E. J. Kramer, X. Li and J. Wang, Phys. Rev. Lett., 2007, 98, 158302.

39 G. Arya and A. Z. Panagiotopoulos, Phys. Rev. Lett., 2005, 95, 188301 .

40 A. Romo-Uribe and A. H. Windle, Macromolecules, 1996, 29, 6246.

41 S. C. Bae, Z. Lin and S. Granick, Macromolecules, 2005, 38, 9275.

42 X. Hao, N. Y. Chan, C. Heck, N. Tanigaki, M. F. Paige, D. E. Dunstan and T. A. Smith, Macromolecules, 2010, 43, 10475. 